\title{
Uses, assessments and perceptions of wildlife: Case study in Loma Bonita, Oaxaca
}

\author{
Ma. Teresa Kido Cruz ${ }^{1 *}$ \\ Tania Zuñiga Marroquín ${ }^{1}$ \\ Antonio Kido $\mathrm{Cruz}^{2}$
}

\begin{abstract}
Even though there is a valid discourse on environmental protection in our country, few efforts have been made in this area, especially when it comes to wildlife. Part of the reason is due to the lack of information and studies on the subject. The objective of this paper is to contribute to our knowledge about the perception of three groups of wild fauna, namely birds, mammals and reptiles in the lower region of the Papaloapan Basin, held by three different groups of the local population: producers, students and university professors. To do this, we used different data analysis techniques: canonical correlation, use indicators and the cost-benefit method. The results yielded 88 identified species, of which 10 lived in the region in the past but are currently reported as non-existent. The most prominent uses are as food and as pets. Among the species with the highest economic value, the crocodile and parrots stand out.
\end{abstract}

Keywords: Perception, use indicators, birds, reptiles, mammals.

\section{Caracterización de la fauna silvestre en Loma Bonita, Oaxaca y su valoración desde la perspectiva local}

\begin{abstract}
Resumen
Aun y cuando el discurso de protección ambiental está vigente en nuestro país, son pocos los esfuerzos que se han realizado en este ámbito, especialmente cuando de fauna silvestre se trata. Parte de la razón obedece a la falta de información y estudios sobre el tema. El presente trabajo tiene como propósito contribuir al conocimiento sobre la percepción de tres grupos de fauna silvestre: aves, mamíferos y reptiles en la región baja de la cuenca del Papaloapan, llevado a cabo por tres diferentes grupos de la población local: productores, estudiantes y profesores universitarios. Para ello se recurrió a distintas técnicas de análisis de datos, la correlación canónica, los indicadores de uso y el método beneficio-costo. Los resultados arrojaron 88 especies identificadas, de las cuales 10 vivieron en el pasado, pero actualmente se reportan como inexistentes. Los usos más destacados son como alimento y como mascotas. Entre las especies con mayor valor económico destacan el cocodrilo y los cotorros.
\end{abstract}

Palabras clave: Percepción, indicadores de uso, aves, reptiles, mamíferos.

\footnotetext{
'Universidad del Papaloapan, Av. Ferrocarril s/n, Ciudad Universitaria, Loma Bonita, Oaxaca.

2Universidad Michoacana de San Nicolás, Facultad de Contaduría y Ciencias Administrativas, Morelia, Michoacán.

*Corresponding author: terekido@hotmail.com
} 


\section{Introduction}

Mexico is one of the few countries with international recognition of its mega diversity. According to the Ministry of the Environment and Natural Resources (Secretaría de Medio Ambiente y Recursos Naturales, 2016), the country has $10 \%$ of the world's recorded species, placing it fourth in the world in amphibians, second in mammals and first in reptiles.

Public demand for certain wildlife species triggers interest in their supply that leads to poaching and illegal trade in them, based mainly on ignorance of the ecological importance of the species, putting habitats and the natural balance at risk.

The success of environmental conservation is often based on the support that the local community can offer to projects of commercial exploitation of both flora and fauna. Bennet and Dearden (2013) argue that perceptions of the impacts experienced by local communities and opinions on the management and governance of protected areas (in this case marine) play a leading role in the implementation of these conservation tools, as they can also have a wide range of positive and negative social, economic, cultural and political impacts on local communities.

It is very likely that local society has an idea, albeit a restricted one, of the importance of wildlife and its relationship to current sustainability and environmental education criteria. Such a perception could be at odds with certain public policies regarding conservation strategies and traditional procedures for the use of natural resources in the region, particularly agriculture and livestock farming.

In this sense, Yang et al. (2015) point out that identifying the main sources of disagreement and understanding the relationships between protected areas and local communities are fundamental for the resolution of conflicts related to the management of protected areas.

Regarding the relationship between ecosystems (genes, species and biodiversity) and people, Davidson-Hunt, Suich, Meijer, and Olsen (2016) point out that assessments should focus on people's use of biodiversity (e.g., food and nutrition, health and medicine, energy, shelter, income, ceremony and trade), recognizing that use is integrated into worldviews that include cultural norms, values

\section{Introducción}

México ha sido uno de los pocos países con reconocimiento internacional sobre su mega diversidad. De acuerdo con la Secretaría de Medio Ambiente y Recursos Naturales (2016), el país cuenta con el $10 \%$ de las especies registradas en el mundo, lo que lo posiciona en el cuarto lugar mundial en anfibios, el segundo en mamíferos y el primero en reptiles.

La demanda de la población por ciertas especies de la fauna silvestre provoca un interés en la oferta que lleva a la caza furtiva y al comercio ilegal de las mismas, basado principalmente en el desconocimiento de la importancia ecológica de las especies, atentando contra los hábitats y el equilibrio natural.

El éxito de la conservación ambiental a menudo se basa en el apoyo que pueda ofrecer la comunidad local a proyectos de explotación comercial tanto de flora como de fauna. Bennet y Dearden (2013), argumentan que las percepciones de los impactos que experimentan las comunidades locales y las opiniones sobre la administración y la gobernanza de áreas protegidas (en este caso marinas), juegan un papel preponderante en la instrumentación de estas herramientas de conservación, ya que también pueden tener una amplia gama de impactos sociales, económicos, culturales y políticos, positivos y negativos en las comunidades locales.

Es muy probable que la sociedad local tenga una idea, -aunque sea restringida- de la importancia que tiene la fauna silvestre y su relación con los actuales criterios de sustentabilidad y educación ambiental. Tal percepción podría estar en discrepancia con ciertas políticas públicas respecto a estrategias de conservación y procedimientos tradicionales de uso de los recursos naturales en la región, particularmente la agricultura y la ganadería.

En este sentido, Yang, et al. (2015) señalan que la identificación de las principales fuentes de desacuerdo y la comprensión de las relaciones entre las áreas protegidas y las comunidades locales son fundamentales para la resolución de conflictos relacionados con la gestión de áreas protegidas.

Respecto a la relación entre los ecosistemas (genes, especies y biodiversidad) y la gente, Davidson-Hunt, Suich, Meijer, y Olsen (2016) señalan que las evaluaciones deben centrarse en el uso de la biodiversidad por parte de las 
and entrenched understandings. The absence of mechanisms for decision-makers to systematically consider the material and symbolic roles that nature plays in people's daily lives can result in interventions or policies that alienate communities or restrict their access to resources. These, in turn, can disrupt traditional land and resource management practices and, as a consequence, undermine livelihoods and threaten critically important species, habitats and ecosystem services, as well as people's relationships with nature.

Thus, it is necessary to implement actions not only in those areas where the original environments have not been significantly altered by human activity or that need to be preserved and restored, but also in communities where wild life cohabits with the local population, not to prohibit the use of species and eradicate customs, but to implement an appropriate system based on the inhabitants' valuation of these elements in the management of protected natural areas and wildlife (Kido \& Seidl, 2008).

Delving into this same idea, Durand and Jiménez (2010) conclude in their work that, "...the current vision of protected natural areas privileges the biologicalecological aspects over the social character of the space. It assumes the existence of a clear distinction between the social and natural spheres, eliminating the possibility of interpreting the environment as a result of the interaction between human beings and the biophysical environment. The denial of the social character of space in protected areas results in the construction of non-places, since local communities lose their referents of space significance and are forced to manage it according to foreign norms, rules and senses..."

Within the above context, it is important to point out that the main objective of this work was to generate comprehensive up-to-date information on the presence-absence status of wildlife species that have been reported for the area, and to know the local population's perception of these species and the economic value they give them. It should be noted that, within the study area, reporting of the presence of wildlife specimens is still relatively common, particularly for certain species that could be in certain protection categories, such as "endangered" or "threatened." This research also personas (por ejemplo, alimentos y nutrición, salud y medicina, energía, refugio, ingresos, ceremonia y comercio), reconociendo que el uso está integrado en visiones del mundo que incluyen normas culturales, valores y entendimientos arraigados. La ausencia de mecanismos para que los tomadores de decisiones consideren sistemáticamente los roles materiales y simbólicos que desempeña la naturaleza en la vida diaria de las personas, puede resultar en intervenciones o políticas que alejan a las comunidades o restrinjan su acceso a los recursos. Estos pueden, a su vez, interrumpir las prácticas tradicionales de manejo de la tierra y los recursos y como consecuencia, socavar los medios de vida y amenazar especies, hábitats y servicios ecosistémicos de importancia crítica, así como las relaciones que las personas tienen con la naturaleza.

De tal suerte, que resulta necesario implementar acciones no solo en aquellas zonas donde los ambientes originales no han sido significativamente alterados por la actividad del ser humano o que requieren ser preservadas y restauradas, sino en las comunidades en donde la fauna silvestre cohabita con la población local, no se trata de prohibir el uso de especies y de erradicar costumbres, sino de implementar un sistema adecuado basado en la valoración misma de los pobladores en el manejo de áreas naturales protegidas y fauna silvestre (Kido \& Seidl, 2008).

Ahondando en esta misma idea, Durand y Jiménez (2010) concluyen en su trabajo que, "... la visión vigente de áreas naturales protegidas privilegia los aspectos biológicos-ecológicos sobre el carácter social del espacio. Se asume la existencia de una distinción clara entre las esferas de lo social y lo natural, eliminando la posibilidad de interpretar al ambiente como resultado de la interacción entre los seres humanos y el entorno biofísico. La negación del carácter social del espacio en áreas protegidas resulta en la construcción de no-lugares, pues las comunidades locales pierden sus referentes de significación del espacio y son obligadas a manejarse en él de acuerdo con normas, reglas y sentidos ajenos..."

Dentro del contexto anterior, es importante señalar que el principal objetivo de este trabajo fue el de generar información integral y actualizada 
generates a wealth of available information that can be used both in academia and for research and use purposes. In the long term, an increase in the degree of perception by the resident population as well as visitors is expected, regarding the concept of wildlife management, biodiversity and environmental education, among others.

\section{Study area and sample determination}

In order to generate a list of the wildlife species that live in and around Loma Bonita, we reviewed the records of the presence-absence of species in the current available literature, which defined three main groups: reptiles, birds and mammals. Subject to other spatial-type delimitations, it was proposed that this region would cover what corresponds to the Lower Papaloapan Basin, assuming that the existing wildlife populations in the region have representatives in Loma Bonita (Torres, 2004). Geographically, the Papaloapan Basin is located between $17^{\circ}$ and $19^{\circ} \mathrm{N}$ and between $95^{\circ}$ and $97^{\circ} \mathrm{W}$. It has an area of $46517 \mathrm{~km}^{2}$ ( $2.4 \%$ of the national territory). Of this area, $51 \%$ corresponds to the state of Oaxaca, $37 \%$ to Veracruz and $12 \%$ to Puebla. The physiographic portion known as the Lower Basin of the Papaloapan River covers approximately $21000 \mathrm{~km}^{2}$ (45 \%), which corresponds to the flat lands of the coastal plain lying below the 100-meter elevation contour line. It is made up of 33 municipalities and land tenure is characterized by owners with ranches of 30 to 100 ha on average, and ejidatarios with small plots (Thiébaut, 2013). According to Hernández-Xolocotzi (1977), the dominant plant communities (disturbed to varying degrees by human activities) in the Lower Papaloapan Basin are: tall evergreen tropical forest, tall deciduous tropical forest, medium-height semievergreen tropical forest, low deciduous tropical forest, flatland oak forest, low inerm tropical forests, evergreen hardwood forest, semi-evergreen scrubland, induced lowland meadows and savannas.

\section{Techniques for gathering and systematizing information}

Questionnaires (surveys), designed to obtain testimonial information from people who have traditionally been in close relationship with wildlife sobre el estatus de presencia-ausencia de las especies de fauna silvestre que han sido reportadas para la zona, así como conocer la percepción y el valor económico que la población local le otorga. Cabe resaltar que, dentro de la zona de estudio, el reporte eventual de la presencia de especímenes de fauna silvestre aún es común, relativamente; de forma particular para ciertas especies que podrían estar en ciertas categorías de protección, como: "en peligro de extinción" o "amenazadas". Como impacto mediato de este trabajo, se obtiene la disponibilidad de un acervo de información, aprovechable tanto en la academia como para fines de investigación y aprovechamiento. A largo plazo, se espera un aumento en el grado de percepción por parte de la población residente además de visitantes, respecto al concepto sobre el manejo de fauna silvestre, biodiversidad y educación ambiental, entre otros.

\section{Área de estudio y determinación de la muestra}

Con el fin de generar un listado de las especies de fauna silvestre que habita dentro y en las inmediaciones de Loma Bonita, se revisaron los registros de la presencia-ausencia de especies en la literatura actual disponible, en la que se definieron tres grupos principales: reptiles, aves y mamíferos. A reserva de otras delimitaciones de tipo espacial, se planteó que dicha región abarcaría lo que corresponde a la cuenca baja del Papaloapan, asumiendo que las poblaciones de fauna silvestre existentes en la región tienen representantes en Loma Bonita (Torres, 2004). Geográficamente, la Cuenca del Papaloapan se localiza entre los $17^{\circ}$ y $19^{\circ} \mathrm{LN}$ y entre los meridianos $95^{\circ}$ y $97^{\circ} \mathrm{LO}$. Cuenta con una superficie de $46517 \mathrm{~km}^{2}$ (2.4 \% de la superficie del territorio nacional). De esta superficie, $51 \%$ corresponde al estado de Oaxaca, $37 \%$ al de Veracruz y $12 \%$ al de Puebla. La porción fisiográfica conocida como Cuenca Baja del Río Papaloapan representa aproximadamente $21000 \mathrm{~km}^{2}$ (45\%), que corresponde a los terrenos planos de la llanura costera que se encuentran abajo de la curva de nivel de los 100 metros de altitud. Está conformada por 33 municipios y la tenencia de la tierra se caracteriza por propietarios con ranchos de 30 a 100 ha en promedio, y ejidatarios con pequeñas parcelas (Thiébaut, 2013). De acuerdo con Hernández-Xolocotzi 
(day laborers, peasants and fishermen), were applied in the municipality of Loma Bonita, Oaxaca. In addition, in order to determine if they represent a benchmark for changing perceptions of wildlife, young people were interviewed, exposed in different ways to current information flows (specifically the Internet), particularizing the sampling to students of the University of Papaloapan. Also, we identified a third group composed of professors-researchers specialized in different disciplines that teach classes in agricultural areas; it should be noted that not all of them are specialty professors, as some also teach core curriculum subjects such as economics, computation or mathematics, to point out some.

The probabilistic sampling technique was used. Consequently, each unit of the population had some probability (other than zero) of being selected (Newbold, Carlson, \& Thome, 2008). The calculation was obtained from the formula for finite populations, suggested by Aguilar-Barojas (2005):

$n=\frac{N Z^{2} p q}{d^{2}(N-1)+Z^{2} p q}$

Where: $\mathrm{n}=$ Sample size; $\mathrm{Z}=$ Confidence level (95 or $99 \%$ ); $p=$ Approximate proportion of the stage phenomenon in the reference population; $\mathrm{q}=$ Proportion of the reference population not represented by the phenomenon studied (1-p); $\mathrm{d}=$ Absolute precision level; $\mathrm{N}=$ Population size.

The estimated universe of the first group was 661 people'. Given a $90 \%$ confidence level and $10 \%$ error, the sample was determined to be 62 producers. The group of students was determined by UNPA's official enrollment in 2016, which was 85 students, yielding a result of 46 surveys; finally, for the group of teachers, 28 were identified by the census, of which 25 agreed to answer it.

'Because there are no official data, the data was obtained from the sum of producers affiliated with the different associations; we used information from the pineapple (a crop of great local importance) growers'association, which has 311 members, the stockbreeders' association, which reported 150 members and 200 fishermen (the most recurrent data in the survey among local fishermen).
(1977), las comunidades vegetales dominantes (perturbadas en diferentes grados por actividades humanas) en la Cuenca Baja del Papaloapan son: selva alta perennifolia, selva alta caducifolia, selva mediana subperennifolia, selva baja caducifolia, bosque planodurifolio, selvas bajas inermes, bosque duriperennifolio, matorral subperennifolio, praderas inducidas de tierras bajas y sabanas.

\section{Técnicas de recopilación y sistematización de la información}

Los cuestionarios (encuestas), diseñados para obtener información testimonial a partir de personas que tradicionalmente han estado en estrecha relación con la fauna silvestre (jornaleros, campesinos y pescadores), fueron aplicados en el municipio de Loma Bonita, Oaxaca. Además, con el fin de determinar si representan un referente de cambio de percepción sobre la fauna silvestre, se entrevistó a personas jóvenes, expuestas de distintas formas a los flujos de información actual (específicamente Internet), particularizando el muestreo a estudiantes de la Universidad del Papaloapan, adicionalmente, se identificó un tercer grupo compuesto por profesores-investigadores especialistas en distintas disciplinas que imparten cátedras a las áreas agropecuarias, cabe señalar que no todos ellos son profesores de especialidad sino que dan clases de tronco común como economía, cómputo o matemáticas, por señalar algunas.

La técnica de muestreo utilizada fue la de tipo probabilístico, de tal manera que cada unidad de la población tuviera alguna probabilidad (diferente de cero) de ser seleccionada (Newbold, Carlson, \& Thome, 2008). El cálculo se obtuvo a partir de la fórmula para poblaciones finitas, sugerida por Aguilar-Barojas (2005):

$n=\frac{N Z^{2} p q}{d^{2}(N-1)+Z^{2} p q}$

Donde: $\mathrm{n}=$ Tamaño de la muestra; $\mathrm{Z}=$ Nivel de confianza (95 o 99 \%); p = Proporción aproximada del fenómeno de estadio en la población referencia; 


\section{Materials and methods}

Methodology for the recognition of the type of wildlife and the perception of it by different generations of local inhabitants

The research was carried out during the period 2016-2018, and consisted of three phases; first, a literature review was carried out where more than 15 sources were consulted, including newspapers, local magazines, specialized journals, books and websites. From this, we created a database of mammal, reptile and bird species reported for the region, which we checked against reports filed by the National Commission for Biodiversity (CONABIO) (2000); the consultation period of these sources spanned 20 years. This resulted in a first list of fauna, which was used to confirm the presence or absence of such fauna in the locality.

Subsequently, we initiated phase two, which consisted of applying surveys to measure the reaction of the three groups, using as a statistical technique the canonical correlation analysis proposed by Hotelling (1935). This technique allows establishing the relationship between predictor variables (IV) and dependent measures (DV); the general form is as follows:

$Y_{1}+Y_{2}+Y_{3}+\cdots+Y_{n}=X_{1}+X_{2}+X_{3}+\cdots+X_{n}$

The base of the correlation is represented by a correlation matrix $(R)$, which is composed of the correlations between the DVs $\left(R_{y y}\right)$, the IVs $\left(R_{x x}\right)$ and between DVs and IVs $\left(R_{x y}\right)$, Badii, Castillo, Cortez, Wong, and Villalpando (2007).

$R=R_{y y}^{-1} R_{y x} R_{x x}^{-1} R_{x y}$

The significance of a canonical correlation is made from the Bartlett test of Wilks' lambda. The lambda varies from 0 to 1 and shows the variance of the error, which is not accounted for by the independent variables. Therefore, the closer the value to 1 means that the independent variables are not accounting for the variance in the dependent variable; and one close to 0 means that the independent variables are accounting for greater variance. Chi square is used to test the significance of lambda. $q$ = Proporción de la población de referencia que no representa el fenómeno estudiado (1-p); $d$ = Nivel de precisión absoluta; $\mathrm{N}=$ Tamaño de la población.

El universo estimado del primer grupo fue de 661 personas ${ }^{1}$, dado un nivel de confianza de $90 \%$ y error de $10 \%$, la muestra se determinó en 62 productores. El grupo de estudiantes estuvo determinado por la matrícula oficial de la UNPA del año 2016 que fue de 85 alumnos, arrojando un resultado de 46 encuestas; finalmente para el grupo de profesores se recurrió al censo de 28 docentes, en la cual 25 accedieron a contestarla.

\section{Materiales y métodos}

Metodología para el reconocimiento del tipo de fauna silvestre y la percepción sobre la misma de diferentes generaciones de pobladores locales

La investigación se llevó a cabo durante el periodo 2016-2018, y constó de tres fases, en un primer momento se realizó una investigación bibliográfica donde se consultaron más de 15 fuentes, incluidos periódicos, revistas locales, revistas especializadas, libros y páginas de internet. A partir de ello se elaboró una base de datos de las especies de mamíferos, reptiles y aves reportadas para la región, que se cotejó con los reportes de la Comisión Nacional para la Biodiversidad (CONABIO) (2000), el periodo de consulta de estas fuentes abarcó 20 años. Con ello se obtuvo un primer listado de la fauna, mismo que se utilizó para confirmar la presencia o ausencia de dicha fauna en la localidad.

Posteriormente, inició la fase dos, que consistió en aplicar encuestas para medir la reacción de los tres grupos, utilizando como técnica estadística, el análisis de correlación canónica propuesta por Hotelling (1935). Esta técnica permite establecer la relación entre variables predictorias $(\mathrm{VI})$ y medidas dependientes (VD), la forma general se presenta como:

$Y_{1}+Y_{2}+Y_{3}+\cdots+Y_{n}=X_{1}+X_{2}+X_{3}+\cdots+X_{n}$

'Debido a que no existen datos oficiales, el dato se obtuvo de la suma de productores adscritos a las diferentes asociaciones; se recurrió a la asociación de piñeros (cultivo de gran importancia local), que cuenta con 311 socios, a la asociación de ganaderos, quienes reportaron 150 agremiados y 200 pescadores (dato más recurrente en el sondeo entre pescadores locales). 


\section{Methodology for assessing the uses of the region's wildlife}

The following indices were analyzed to calculate the use value that inhabitants confer on wildlife species: the cultural importance index (CII) and the specific use value index (SUVI). The first was suggested by Turner (1988), modified by Figueroa-Solano (2000) and used by several authors; Jiménez-Ferrer, VelascoPérez, Uribe-Gómez, and Soto-Pinto (2008), Suárez, Palacios, Razo, Araujo, and Goche (2011) and ÁvilaNájera, Rosas-Rosas, Tarango-Arámbula, MartínezMontoya, and Santoyo-Brito (2011), among others. The second was proposed by Phillips and Gentry (1993) and subsequently used by authors such as Alexiades (1996) and Puc and Retana (2012). Taking both proposals into account, the valuation of uses was determined from the following indices:

$G U I=S U / S U C$

Where: GUI is the General Use Index, SU corresponds to the number of uses per species and SUC represents the total number of specific use categories.

$F E U_{x}=M V U E_{x} / E V U$

Where: FEU is the Frequency of Economic Use for the species $x$, MVSU represents the number of mentions for the species $x$ with market value and $N$ represents the total number of respondents.

$U V_{j x}={ }^{M U_{j x}} / M T U_{j}$

Where: UV is the Use Value $j$ for species $x, M U$ represents the number of mentions of use $\boldsymbol{j}$ for species $x$ and MTU corresponds to mentions of use $j$ for all species.

\section{Economic valuation methodology}

Finally, in a third stage, those people who declared some use of wildlife were identified and located for in-depth interviews. With the information obtained, the economic valuation of the species was made. The economic value of a good or service is measured by the sum of the intentions of many individuals to pay for the protection or the opportunity cost of the good or service-product. This means that the intention to
La base de la correlación está representada por una matriz de correlación $(R)$, misma que se compone de las correlaciones entre las VDs $\left(R_{y y}\right)$, las Vis $\left(R_{x x}\right)$ y entre VDs y Vis $\left(R_{x y}\right)$, Badii, Castillo, Cortez, Wong, y Villalpando (2007).

$R=R_{y y}^{-1} R_{y x} R_{x x}^{-1} R_{x y}$

La significancia de una correlación canónica se realiza a partir de la prueba de Bartlett de la lambda de Wilks. La lambda varía de 0 a 1 y muestra la varianza del error, la que no se contabiliza por las variables independientes. Por lo que entre más cercano sea el valor a 1 significa que las variables independientes no están contabilizando la varianza en la variable dependiente; y uno cercano a 0 , significa que las variables independientes están contabilizando mayor varianza. Para probar la significancia de lambda se usa chi cuadrada.

\section{Metodología para valorar los usos de la fauna silvestre de la región}

Para calcular el valor de uso que los pobladores confieren a las especies faunísticas se analizaron los siguientes índices; el índice de importancia cultural (IIC) y el índice de valor de uso específico (VUEis). El primero fue sugerido por Turner (1988), modificado por Figueroa-Solano (2000) y utilizado por diversos autores; Jiménez-Ferrer, Velasco-Pérez, Uribe-Gómez, y Soto-Pinto (2008), Suárez, Palacios, Razo, Araujo, y Goche (2011) y Ávila-Nájera, Rosas-Rosas, TarangoArámbula, Martínez-Montoya, y Santoyo-Brito (2011) entre otros. El segundo fue propuesto por Phillips y Gentry (1993) y utilizado posteriormente por autores tales como Alexiades (1996) y Puc y Retana (2012). Tomando como base ambas propuestas, la valoración de usos se determinó a partir de los siguientes índices:

$I G U=U E / C U E$

Donde: IGU es el Índice General de Uso, UE corresponde al número de usos por especie y CUE representa el total de categorías de uso determinadas.

$F U E_{x}=M V U E_{x} / E V U$ 
pay reflects individual preferences in relation to the given good or product. Thus, economic valuation in the environmental context is the measure of people's preferences in monetary terms for a natural good. The use of monetary values is very important because it allows comparing environmental values with development values. Economic valuation is essentially about discovering the values that human beings give to the environment. Normally, society values things according to their usefulness, that is, it gives them real or economic value, usually expressed in money. It can be market value, commercial value or non-commercial value.

Knowing this value is fundamental to the environmental management proposal. For its calculation, we used the direct valuation method called production function, which is derived from the cost-benefit analysis. Dixon, Scura, Carpenter, and Sherman (1994) define the method as a technique for analyzing the stocks and flows of benefits and costs over a period, and is based on individual preferences, willingness to pay, and social welfare. Individual preferences are a basis for measuring benefits (Pearce \& Turner, 1990; Ruitenbeek, 1990).

\section{Results}

Results on local recognition of reptiles, birds and mammals

According to SEMARNAT (2009), Mexican law defines wildlife as "resident or migratory invertebrate and vertebrate animals that live in natural conditions in the national territory and that do not require the care of man for their survival." Based on this criterion, the literature review provided a total of 88 species located in the region, of which 36 belong to the bird group, 23 to the mammal group and 29 to the reptile one (Table 1).

It should be noted that of the 88 species identified in the literature, 78 are still present in the region, and 10 existed in the past. From the group of birds: roseate spoonbill and wood stork; from the group of reptiles: indigo snake, five-striped snake and blunthead tree snake, and from the group of mammals: ocelot, collared peccary, Central American red brocket, margay and Mexican black agouti. Locally extinct species represent $9 \%$ of the total.
Donde: FUE es la Frecuencia de Uso Económico para la especie $x, M V U E$ representa el número de menciones para la especie $x$ con valor de mercado $y$ $N$ representa el número total de informantes

$$
V U_{j x}={ }^{M U_{j x}} / M T U_{j}
$$

Donde: $V U$ es el Valor del Uso $j$ para la especie $x, M U$ representa el número de menciones del uso $j$ para la especie x y MTU corresponde a las menciones del uso j para todas las especies.

\section{Metodología de valoración económica}

Finalmente, en una tercera etapa se identificaron aquellas personas que declararon algún uso de la fauna silvestre y se les localizó para realizar entrevistas a profundidad. Con la información obtenida se hizo la valoración económica de las especies. El valor económico de un bien o servicio es medido por la suma de las intenciones por pagar de muchos individuos por la protección o su costo de oportunidad del bien o servicio-producto. Esto significa que la intención por pagar refleja las preferencias individuales del determinado bien o producto. Entonces, la valoración económica en el contexto medio ambiental es la medida de las preferencias de la gente en términos monetarios por un bien natural. El uso de valores monetarios es muy importante porque permite comparar valores medio ambientales con valores de desarrollo. La valoración económica trata esencialmente de descubrir los valores que los seres humanos dan al medio ambiente. Normalmente, la sociedad valora las cosas de acuerdo con su utilidad, es decir, le otorga un valor real o económico, expresado generalmente en dinero. Puede ser valor de mercado, valor comercial o valor no comercial.

El conocer este valor resulta fundamental en la propuesta de manejo ambiental. Para su cálculo se recurrió al método de valoración directa denominado función de producción, que se deriva del análisis costo-beneficio. Dixon, Scura, Carpenter, y Sherman (1994) definen el método como una técnica de análisis de existencias y flujos de beneficios y costos en un periodo, y se basa en las preferencias individuales, la disponibilidad a pagar y el bienestar social. Las preferencias individuales son 
Table 1. List of fauna of the Papaloapan region.

Cuadro 1. Lista de fauna de la región del Papaloapan.

\begin{tabular}{|c|c|c|c|c|c|}
\hline $\begin{array}{l}\text { Common name / } \\
\text { Nombre común }\end{array}$ & $\begin{array}{c}\text { Scientific name / } \\
\text { Nombre } \\
\text { científico }\end{array}$ & $\begin{array}{l}\text { GROUP / } \\
\text { GRUPO }\end{array}$ & $\begin{array}{l}\text { Common name / } \\
\text { Nombre común }\end{array}$ & $\begin{array}{c}\text { Scientific name / } \\
\text { Nombre } \\
\text { científico }\end{array}$ & $\begin{array}{l}\text { GROUP / } \\
\text { GRUPO }\end{array}$ \\
\hline $\begin{array}{l}\text { Golden-fronted } \\
\text { woodpecker / } \\
\text { Pájaro carpintero }\end{array}$ & $\begin{array}{l}\text { Meleanerpes } \\
\text { aurifroms }\end{array}$ & Birds / Aves & $\begin{array}{l}\text { Neotropical river } \\
\text { otter / } \\
\text { Nutria de río/perro } \\
\text { de agua }\end{array}$ & Lontra longicaudis & $\begin{array}{l}\text { Mammals / } \\
\text { Mamíferos }\end{array}$ \\
\hline $\begin{array}{l}\text { Great kiskadee / } \\
\text { Pecho amarillo }\end{array}$ & $\begin{array}{l}\text { Pitangus } \\
\text { sulphuratus }\end{array}$ & Birds / Aves & $\begin{array}{l}\text { Mexican long- } \\
\text { tongued bat / } \\
\text { Murciélago } \\
\text { trompudo }\end{array}$ & $\begin{array}{l}\text { Choeronycteris } \\
\text { mexicana }\end{array}$ & $\begin{array}{l}\text { Mammals / } \\
\text { Mamíferos }\end{array}$ \\
\hline $\begin{array}{l}\text { Blue-gray } \\
\text { tanager / } \\
\text { Azulejo }\end{array}$ & Thraupis episcopus & Birds / Aves & $\begin{array}{l}\text { Mexican cottontail / } \\
\text { Conejo silvestre o } \\
\text { montés }\end{array}$ & $\begin{array}{l}\text { Sylvilagus } \\
\text { cunicularius }\end{array}$ & $\begin{array}{l}\text { Mammals / } \\
\text { Mamíferos }\end{array}$ \\
\hline $\begin{array}{l}\text { Mexican grackle / } \\
\text { Picho }\end{array}$ & $\begin{array}{l}\text { Quiscalus } \\
\text { mexicanus }\end{array}$ & Birds / Aves & Jaguarundi / Onza & $\begin{array}{l}\text { Puma } \\
\text { yagouaroundi }\end{array}$ & $\begin{array}{l}\text { Mammals / } \\
\text { Mamíferos }\end{array}$ \\
\hline $\begin{array}{l}\text { Buff-bellied } \\
\text { hummingbird / } \\
\text { Colibrí }\end{array}$ & $\begin{array}{l}\text { Amazilia } \\
\text { yucatanensis }\end{array}$ & Birds / Aves & $\begin{array}{l}\text { Mexican hairy dwarf } \\
\text { porcupine / } \\
\text { Puercoespín }\end{array}$ & $\begin{array}{l}\text { Sphiggurus } \\
\text { mexicanus }\end{array}$ & $\begin{array}{l}\text { Mammals / } \\
\text { Mamíferos }\end{array}$ \\
\hline $\begin{array}{l}\text { Great egret / } \\
\text { Garza blanca }\end{array}$ & Ardea alba & Birds / Aves & $\begin{array}{l}\text { White-nosed coati / } \\
\text { Coatí- tejón solitario }\end{array}$ & Nasua narica & $\begin{array}{l}\text { Mammals / } \\
\text { Mamíferos }\end{array}$ \\
\hline $\begin{array}{l}\text { Ferruginous pygmy } \\
\text { owl / } \\
\text { Tecolote }\end{array}$ & $\begin{array}{l}\text { Glaucidium } \\
\text { brasilianum }\end{array}$ & Birds / Aves & $\begin{array}{l}\text { Nine-banded } \\
\text { armadillo / } \\
\text { Armadillo - toche }\end{array}$ & $\begin{array}{l}\text { Dasypus } \\
\text { novemcinctus }\end{array}$ & $\begin{array}{l}\text { Mammals / } \\
\text { Mamíferos }\end{array}$ \\
\hline $\begin{array}{l}\text { Black-bellied } \\
\text { whistling duck / } \\
\text { Pichiche }\end{array}$ & $\begin{array}{l}\text { Dendrocygna } \\
\text { autumnalis }\end{array}$ & Birds / Aves & $\begin{array}{l}\text { Bobcat / } \\
\text { Gato montés }\end{array}$ & Lynx rufus & $\begin{array}{l}\text { Mammals / } \\
\text { Mamíferos }\end{array}$ \\
\hline $\begin{array}{l}\text { Red-billed } \\
\text { pigeon / } \\
\text { Paloma mora }\end{array}$ & $\begin{array}{l}\text { Patagioenas } \\
\text { flavirostris }\end{array}$ & Birds / Aves & Coyote & $\begin{array}{l}\text { Canis latrans Say, } \\
1823\end{array}$ & $\begin{array}{l}\text { Mammals / } \\
\text { Mamíferos }\end{array}$ \\
\hline $\begin{array}{l}\text { Hooded warbler / } \\
\text { Chipe } \\
\text { encapuchado }\end{array}$ & Wilsonia citrina & Birds / Aves & $\begin{array}{l}\text { Southern pocket } \\
\text { gopher / } \\
\text { Tuza }\end{array}$ & $\begin{array}{l}\text { Thomomys } \\
\text { umbrinus }\end{array}$ & $\begin{array}{l}\text { Mammals / } \\
\text { Mamíferos }\end{array}$ \\
\hline $\begin{array}{l}\text { Red-lored } \\
\text { amazon / } \\
\text { Cotorro }\end{array}$ & $\begin{array}{l}\text { Amazona } \\
\text { autumnalis }\end{array}$ & Birds / Aves & $\begin{array}{l}\text { Mexican black } \\
\text { agouti / } \\
\text { Agouti negro }\end{array}$ & $\begin{array}{l}\text { Dasyprocta } \\
\text { mexicana }\end{array}$ & $\begin{array}{l}\text { Mammals / } \\
\text { Mamíferos }\end{array}$ \\
\hline Pepencha & $\begin{array}{l}\text { Columbina } \\
\text { talpacoti }\end{array}$ & Birds / Aves & $\begin{array}{l}\text { Lowland paca / } \\
\text { Tepezcuintle }\end{array}$ & Caniculus paca & $\begin{array}{l}\text { Mammals / } \\
\text { Mamíferos }\end{array}$ \\
\hline $\begin{array}{l}\text { Cooper's hawk / } \\
\text { Gavilán }\end{array}$ & Accipiter cooperii & Birds / Aves & Ocelot / Ocelote & Leopardus pardalis & $\begin{array}{l}\text { Mammals / } \\
\text { Mamíferos }\end{array}$ \\
\hline $\begin{array}{l}\text { Black vulture / } \\
\text { Zopilote }\end{array}$ & Coragyps atratus & Birds / Aves & Margay / Tigrillo & Leopardus wiedii & $\begin{array}{l}\text { Mammals / } \\
\text { Mamíferos }\end{array}$ \\
\hline $\begin{array}{l}\text { Common ground } \\
\text { dove / } \\
\text { Tórtola coquita }\end{array}$ & $\begin{array}{l}\text { Columbina } \\
\text { passerina }\end{array}$ & Birds / Aves & $\begin{array}{l}\text { Collared } \\
\text { peccary / } \\
\text { Pecarí de collar }\end{array}$ & Pecari tajacu & $\begin{array}{l}\text { Mammals / } \\
\text { Mamíferos }\end{array}$ \\
\hline $\begin{array}{l}\text { Inca dove / } \\
\text { Tórtola cola larga }\end{array}$ & Columbina inca & Birds / Aves & $\begin{array}{l}\text { Banded arboreal } \\
\text { alligator lizard / } \\
\text { Lagarto alicate }\end{array}$ & Abronia taeniata & Reptiles \\
\hline
\end{tabular}


Table 1. List of fauna of the Papaloapan region. ( cont.) Cuadro 1. Lista de fauna de la región del Papaloapan. ( cont.)

\begin{tabular}{|c|c|c|c|c|c|}
\hline $\begin{array}{l}\text { Common name / } \\
\text { Nombre común }\end{array}$ & $\begin{array}{c}\text { Scientific name / } \\
\text { Nombre } \\
\text { científico }\end{array}$ & $\begin{array}{l}\text { GROUP / } \\
\text { GRUPO }\end{array}$ & $\begin{array}{l}\text { Common name / } \\
\text { Nombre común }\end{array}$ & $\begin{array}{c}\text { Scientific name / } \\
\text { Nombre } \\
\text { científico }\end{array}$ & $\begin{array}{l}\text { GROUP / } \\
\text { GRUPO }\end{array}$ \\
\hline $\begin{array}{l}\text { Ruddy ground- } \\
\text { dove / } \\
\text { Tórtola rojiza }\end{array}$ & $\begin{array}{l}\text { Columbina } \\
\text { talpacoti }\end{array}$ & Birds / Aves & $\begin{array}{l}\text { Rainbow ameiva / } \\
\text { Lagarto arcoíris }\end{array}$ & Ameiva undulata & Reptiles \\
\hline $\begin{array}{l}\text { White-winged } \\
\text { dove / } \\
\text { Paloma blanca }\end{array}$ & Zenaida asiática & Birds / Aves & $\begin{array}{l}\text { Silky anole / } \\
\text { Lagartija escamosa }\end{array}$ & Anolis sericeus & Reptiles \\
\hline $\begin{array}{l}\text { Mourning dove / } \\
\text { Paloma huilota }\end{array}$ & Zenaida macroura & Birds / Aves & $\begin{array}{l}\text { Blackbelly } \\
\text { racerunner / } \\
\text { Lagartija siete líneas }\end{array}$ & Aspidoscelis deppei & Reptiles \\
\hline $\begin{array}{l}\text { Olive-throated } \\
\text { parakeet / } \\
\text { Cotorritos }\end{array}$ & Aratinga nana & Birds / Aves & $\begin{array}{l}\text { Rosebelly lizard / } \\
\text { Lagartija escamosa } \\
\text { rosada }\end{array}$ & $\begin{array}{l}\text { Sceloporus } \\
\text { variabilis }\end{array}$ & Reptiles \\
\hline $\begin{array}{l}\text { Northern } \\
\text { bobwhite / } \\
\text { Codorniz cotuí }\end{array}$ & Colinus virginianus & Birds / Aves & $\begin{array}{l}\text { Brown forest skink / } \\
\text { Lagartija parda }\end{array}$ & $\begin{array}{l}\text { Sphenomorphus } \\
\text { cherriei }\end{array}$ & Reptiles \\
\hline $\begin{array}{l}\text { Western cattle } \\
\text { egret / } \\
\text { Garza ganadera }\end{array}$ & Bubulcus ibis & Birds / Aves & $\begin{array}{l}\text { Broadfoot } \\
\text { mushroomtongue } \\
\text { salamander / } \\
\text { Salamandra }\end{array}$ & $\begin{array}{l}\text { Bolitoglossa } \\
\text { platydactyla }\end{array}$ & Reptiles \\
\hline $\begin{array}{l}\text { Anhinga / } \\
\text { Pato aguja }\end{array}$ & Anhinga anhinga & Birds / Aves & $\begin{array}{l}\text { Morelet's crocodile / } \\
\text { Cocodrilo }\end{array}$ & $\begin{array}{l}\text { Crocodylus } \\
\text { moreletii }\end{array}$ & Reptiles \\
\hline $\begin{array}{l}\text { Great blue heron / } \\
\text { Garza azul }\end{array}$ & Ardea herodias & Birds / Aves & $\begin{array}{l}\text { White-lipped mud } \\
\text { turtle / } \\
\text { Tortuga casquito }\end{array}$ & $\begin{array}{l}\text { Kinosternon } \\
\text { leucostomum }\end{array}$ & Reptiles \\
\hline $\begin{array}{l}\text { Roadside hawk / } \\
\text { Aguililla caminera }\end{array}$ & Buteo magnirostris & Birds / Aves & $\begin{array}{l}\text { Caninana / } \\
\text { Tigra cazadora }\end{array}$ & Spilotes pullatus & Reptiles \\
\hline $\begin{array}{l}\text { Green heron / } \\
\text { Garza verde }\end{array}$ & Butorides virescens & Birds / Aves & $\begin{array}{l}\text { Black blind snake / } \\
\text { Serpiente } \\
\text { hormiguera }\end{array}$ & Epictia goudoti & Reptiles \\
\hline $\begin{array}{l}\text { Common } \\
\text { nighthawk / } \\
\text { Tapacaminos }\end{array}$ & Chordeiles minor & Birds / Aves & $\begin{array}{l}\text { Narrow-bridged } \\
\text { musk turtle / } \\
\text { Tortuga chopontil }\end{array}$ & $\begin{array}{l}\text { Claudius } \\
\text { angustatus }\end{array}$ & Reptiles \\
\hline $\begin{array}{l}\text { Band-backed } \\
\text { wren / } \\
\text { Matraca }\end{array}$ & $\begin{array}{l}\text { Campylorhynchus } \\
\text { zonatus }\end{array}$ & Birds / Aves & $\begin{array}{l}\text { Meso-American } \\
\text { slider / } \\
\text { Tortuga pinta }\end{array}$ & $\begin{array}{l}\text { Trachemys scripta } \\
\text { venusta }\end{array}$ & Reptiles \\
\hline $\begin{array}{l}\text { Baltimore oriole / } \\
\text { Oropéndola }\end{array}$ & Icterus galbula & Birds / Aves & $\begin{array}{l}\text { Mexican musk turtle / } \\
\text { Tortuga tres lomos }\end{array}$ & $\begin{array}{l}\text { Staurotypus } \\
\text { triporcatus }\end{array}$ & Reptiles \\
\hline $\begin{array}{l}\text { Northern jacana / } \\
\text { Jacana }\end{array}$ & Jacana spinosa & Birds / Aves & $\begin{array}{l}\text { Red-eared slider / } \\
\text { Tortuga pinta orejas } \\
\text { rojas }\end{array}$ & $\begin{array}{l}\text { Trachemys scripta } \\
\text { elegans }\end{array}$ & Reptiles \\
\hline $\begin{array}{l}\text { Ringed kingfisher / } \\
\text { Martín pescador de } \\
\text { collar }\end{array}$ & $\begin{array}{l}\text { Megaceryle } \\
\text { torquata }\end{array}$ & Birds / Aves & $\begin{array}{l}\text { Brown basilisk / } \\
\text { Teterete }\end{array}$ & Basiliscus vittatus & Reptiles \\
\hline $\begin{array}{l}\text { Wood stork / } \\
\text { Cigüeña }\end{array}$ & $\begin{array}{l}\text { Mycteria } \\
\text { americana }\end{array}$ & Birds / Aves & $\begin{array}{l}\text { Mexican Racerunner / } \\
\text { Lagartija corredora }\end{array}$ & $\begin{array}{l}\text { Cnemidophorus } \\
\text { guttatus }\end{array}$ & Reptiles \\
\hline
\end{tabular}


Table 1. List of fauna of the Papaloapan region. ( cont.) Cuadro 1. Lista de fauna de la región del Papaloapan. ( cont.)

\begin{tabular}{|c|c|c|c|c|c|}
\hline $\begin{array}{l}\text { Common name / } \\
\text { Nombre común }\end{array}$ & $\begin{array}{c}\text { Scientific name / } \\
\text { Nombre } \\
\text { científico }\end{array}$ & $\begin{array}{l}\text { GROUP / } \\
\text { GRUPO }\end{array}$ & $\begin{array}{l}\text { Common name / } \\
\text { Nombre común }\end{array}$ & $\begin{array}{c}\text { Scientific name / } \\
\text { Nombre } \\
\text { científico }\end{array}$ & $\begin{array}{l}\text { GROUP / } \\
\text { GRUPO }\end{array}$ \\
\hline $\begin{array}{l}\text { Roseate spoonbill / } \\
\text { Espátula rosada }\end{array}$ & Platalea ajaja & Birds / Aves & $\begin{array}{l}\text { Green iguana / } \\
\text { Iguana }\end{array}$ & Iguana iguana & Reptiles \\
\hline $\begin{array}{l}\text { Tropical kingbird / } \\
\text { Tirano }\end{array}$ & $\begin{array}{l}\text { Tyrannus } \\
\text { melancholicus }\end{array}$ & Birds / Aves & $\begin{array}{l}\text { Common snapping } \\
\text { turtle / } \\
\text { Tortuga lagarto }\end{array}$ & $\begin{array}{l}\text { Chelydra } \\
\text { serpentina }\end{array}$ & Reptiles \\
\hline $\begin{array}{l}\text { Clay-colored } \\
\text { thrush / } \\
\text { Primavera }\end{array}$ & Turdus grayi & Birds / Aves & $\begin{array}{l}\text { Mexican spiny-tailed } \\
\text { iguana / } \\
\text { Iguana negra, } \\
\text { garrobo }\end{array}$ & $\begin{array}{l}\text { Ctenosaura } \\
\text { pectinata }\end{array}$ & Reptiles \\
\hline $\begin{array}{l}\text { Fork-tailed } \\
\text { flycatcher / } \\
\text { Tijereta sabanera }\end{array}$ & Tyrannus savana & Birds / Aves & $\begin{array}{l}\text { Northern boa / } \\
\text { Boa, Mazacoa }\end{array}$ & $\begin{array}{l}\text { Boa constrictor } \\
\text { imperator }\end{array}$ & Reptiles \\
\hline $\begin{array}{l}\text { Hooded skunk / } \\
\text { Zorrillo }\end{array}$ & Mephitis macroura & $\begin{array}{l}\text { Mammals / } \\
\text { Mamíferos }\end{array}$ & $\begin{array}{l}\text { Black-striped } \\
\text { snake / } \\
\text { Culebra de rayas } \\
\text { negras }\end{array}$ & $\begin{array}{l}\text { Coniophanes } \\
\text { imperialis }\end{array}$ & Reptiles \\
\hline $\begin{array}{l}\text { Tayra / } \\
\text { Cabeza de viejo }\end{array}$ & Eira barbara & $\begin{array}{l}\text { Mammals / } \\
\text { Mamíferos }\end{array}$ & $\begin{array}{l}\text { Five-striped snake / } \\
\text { Culebra de vientre }\end{array}$ & $\begin{array}{l}\text { Coniophanes } \\
\text { quinquevittatus }\end{array}$ & Reptiles \\
\hline $\begin{array}{l}\text { Kinkajou / } \\
\text { Martucha }\end{array}$ & Potos flavus & $\begin{array}{l}\text { Mammals / } \\
\text { Mamíferos }\end{array}$ & $\begin{array}{l}\text { Indigo snake / } \\
\text { Culebra índigo }\end{array}$ & Drymarchon corais & Reptiles \\
\hline $\begin{array}{l}\text { Mexican gray } \\
\text { squirrel / } \\
\text { Ardilla gris vientre } \\
\text { rojo }\end{array}$ & Sciurus aureogaster & $\begin{array}{l}\text { Mammals / } \\
\text { Mamíferos }\end{array}$ & $\begin{array}{l}\text { Blunthead tree } \\
\text { snake / } \\
\text { Culebra cordelilla } \\
\text { chata }\end{array}$ & Imantodes cenchoa & Reptiles \\
\hline $\begin{array}{l}\text { Virginia opossum / } \\
\text { Tlacuache }\end{array}$ & $\begin{array}{l}\text { Didelphis } \\
\text { virginiana }\end{array}$ & $\begin{array}{l}\text { Mammals / } \\
\text { Mamíferos }\end{array}$ & $\begin{array}{l}\text { Neotropical whip } \\
\text { snake / } \\
\text { Culebra chirriadora }\end{array}$ & $\begin{array}{l}\text { Masticophis } \\
\text { mentovarius }\end{array}$ & Reptiles \\
\hline $\begin{array}{l}\text { Northern } \\
\text { tamandua / } \\
\text { Oso hormiguero- } \\
\text { brazo fuerte }\end{array}$ & $\begin{array}{l}\text { Tamandua } \\
\text { mexicana }\end{array}$ & $\begin{array}{l}\text { Mammals / } \\
\text { Mamíferos }\end{array}$ & $\begin{array}{l}\text { Diamondback water } \\
\text { snake / } \\
\text { Culebra de agua de } \\
\text { diamantes }\end{array}$ & Nerodia rhombifer & Reptiles \\
\hline $\begin{array}{l}\text { Raccoon / } \\
\text { Mapache }\end{array}$ & Procyon lotor & $\begin{array}{l}\text { Mammals / } \\
\text { Mamíferos }\end{array}$ & $\begin{array}{l}\text { Western ribbon } \\
\text { snake / } \\
\text { Culebra listonada }\end{array}$ & $\begin{array}{l}\text { Thamnophis } \\
\text { proximus }\end{array}$ & Reptiles \\
\hline $\begin{array}{l}\text { Central American } \\
\text { red brocket / } \\
\text { Venado temazate }\end{array}$ & Mazama temama & $\begin{array}{l}\text { Mammals / } \\
\text { Mamíferos }\end{array}$ & $\begin{array}{l}\text { Fer-de-lance / } \\
\text { Sorda, Nauyaca }\end{array}$ & Bothrops asper & Reptiles \\
\hline
\end{tabular}

Sources / Fuente: Adams, et al., (2008); Alfaro, García-García, and Santos-Moreno (2006); Álvarez (2003); Bezaury-Creel, Gutiérrez-Carbonell, and Remolina (2009); Boege (2009); Buena-Zepeda, Castillo-Romano, Castillo-Campos, Pale-Pale, and Moreno-Casasola (2012); Campbell (1998); Casas-Andreu, Méndez de la Cruz, and Camarillo (1996); Cruz-Espinoza, González-Pérez, and Ronel-Vázquez (2012); Flores-Villela (1993); Gese, Bekoff, Andelt, Carbyn, and Knowlton (2008); Gómez-Nísino (2006); Naturalista (2016); Pérez-Higadera and Smith (1991); Pérez-Irineo and Santos Moreni (2012); Pérez-Lustre, Contreras-Díaz, and Santos-Moreno (2006); Ramírez-González, LaraBrenis, and Mijangos-Arrazola (2014); Ruiz-Velásquez, Andrés-Reyes, and Santos-Moreno (2014); Sánchez-Herrera, López-Segurajauregui, García-Naranjo, and Benitez-Díaz (2011); Smith, H. and Smith, R. (1976); Tamayo (1977); World Wildlife Fund (2000); Zertuche (1981). 
In order to determine the degree of recognition of the species of the three different groups studied, central tendency measures were used. The results show a high degree of recognition for the group of producers, followed by the professors and lastly the students who were the least able to identify the species (Table 2).

In an attempt to get an idea of the abundance of the species, respondents were asked about their perception of the estimated quantities of each species. The scale was determined in four values, ranging from high to very low; of the total number of specimens, only $14 \%$ recorded a high abundance, in the case of reptiles $42 \%$ are reported as scarce and $17 \%$ of mammals are perceived as very scarce, in addition to the fact that this group has the greatest reported scarcity. Table 3 presents greater detail of the results by group. una base de medición de los beneficios (Pearce \& Turner, 1990; Ruitenbeek, 1990).

\section{Resultados}

Resultados sobre el reconocimiento local de reptiles, aves y mamíferos

De acuerdo con la SEMARNAT (2009), la ley en México define la vida silvestre como "animales invertebrados y vertebrados residentes o migratorios, que viven en condiciones naturales en el territorio nacional y que no requieren del cuidado del hombre para su supervivencia". Atendiendo este criterio, la revisión bibliográfica proporcionó un total de 88 especies que se localizan en la región, de las cuales 36 pertenecen al grupo de las aves, 23 a los mamíferos y 29 a los reptiles (Cuadro 1).

Table 2. Absolute and relative frequency measurements by study group.

Cuadro 2. Medidas de frecuencia absoluta y relativa por grupo de estudio.

\begin{tabular}{cccccccc}
\hline $\begin{array}{c}\text { Group / } \\
\text { Grupo }\end{array}$ & $\begin{array}{c}\text { Range } \\
\text { Rango }\end{array}$ & \multicolumn{2}{c}{ Producers / Productor } & \multicolumn{2}{c}{ Students / Estudiantes } & \multicolumn{2}{c}{ Professors / Profesores } \\
\hline \multirow{3}{*}{ Birds / Aves } & & $\mathrm{n}_{\mathrm{i}}$ & $\mathrm{f}_{\mathrm{i}}$ & $\mathrm{n}_{\mathrm{i}}$ & $\mathrm{f}_{\mathrm{i}}$ & $\mathrm{n}_{\mathrm{i}}$ & $\mathrm{f}_{\mathrm{i}}$ \\
& $(30-34)$ & 60 & 0.90 & 20 & 0.43 & 5 & 0.20 \\
& $(25-29)$ & 6 & 0.10 & 12 & 0.26 & 12 & 0.48 \\
& $(20-24)$ & & & 14 & 0.31 & 8 & 0.32 \\
& $(16-18)$ & 61 & 0.92 & 18 & 0.39 & 12 & 0.48 \\
Mammals / & $(12-15)$ & 5 & 0.08 & 22 & 0.48 & 8 & 0.32 \\
Mamíferos & $(8-11)$ & & & 6 & 0.13 & 5 & 0.20 \\
Reptiles & $(22-26)$ & 66 & 1.00 & 15 & 0.33 & 17 & 0.68 \\
\hline
\end{tabular}

Source: Author-made.

Fuente: Elaboración propia.

Table 3. Perception of the abundance of species under study.

Cuadro 3. Percepción de la abundancia de las especies en estudio.

\begin{tabular}{cccccc}
\hline & N & A lot / Mucho & Regular & Little / Poco & $\begin{array}{c}\text { Very little / } \\
\text { Muy Poco }\end{array}$ \\
\hline Birds / Aves & 34 & 9 & 15 & 7 & 3 \\
Mammals / Mamíferos & 18 & 3 & 3 & 8 & 4 \\
Reptiles & 26 & 1 & 12 & 27 & 1 \\
Total & 78 & 13 & 30 & & 8 \\
\hline
\end{tabular}

Source: Author-made.

Fuente: Elaboración propia. 
According to Santos-Fita, Argueta-Villamar, Astorga-Domínguez, and Quiñonez-Martínez (2012), the cognitive, emotional, and behavioral bonds established between human beings and the surrounding fauna define man himself, either by the tangible value of the latter or by its ecological relevance. It is therefore necessary to know not only the perception, but also the relationship that develops and the attitudes towards the different species. In this project, respondents were questioned about their reaction to the different specimens identified; the results for some of them, especially for most mammals, was indifferent in the three groups surveyed. However, there are significant differences in the reaction of the different groups towards some specimens of the bird and reptile groups, so a correspondence analysis was performed. The results show an association between the behavior and the group to which they belong (Chi-square test, $P<0.0001$ ), with producers making the greatest use of wildlife (Figures 1 and 2).
Cabe destacar que de las 88 especies identificadas en la bibliografía: 78 aún tienen presencia en la región, 10 existieron en el pasado. Del grupo de aves: espátula rosada y cigüeña; del de reptiles: culebra índigo, culebra de vientre punteado y culebra cordelilla chata, y del de mamíferos: ocelote, pecarí de collar, venado temazate, tigrillo y Agouti negro. Las especies extintas localmente representan $9 \%$ del total.

Para poder determinar el grado de reconocimiento de las especies de los tres diferentes grupos estudiados, se recurrió a las medidas de tendencia central. Los resultados muestran un alto grado de reconocimiento para el grupo de productores, seguido por los profesores y con menor identificación de especies están los estudiantes. Cuadro 2.

Con la intención de obtener una idea de la abundancia de las especies, se preguntó a los encuestados sobre su percepción en cuanto a las cantidades estimadas de cada una de las especies. La

Figura 1. Bird correspondence analysis.

Figura 1. Análisis de correspondencia aves.




Figure 2. Reptile correspondence analysis.

Figura 2. Análisis de correspondencia reptiles.

\section{CCA Map / Symmetric}

(axes F1 and F2: 97.31 \%)



Source: Author-made.

Fuente: Elaboración propia.

\section{Results on use value indicators}

When presented as satisfiers of human needs, natural resources become useful elements, understood as utility or use, those activities through which human beings obtain some benefit, gain, fruit or enjoyment of an animal population. The uses may be direct or indirect, consumptive or non-consumptive (Alcérreca, et al., 1988). The activities may be personal or family (when used for family consumption), commercial (when exchanged in a market) or of another nature such as education and scientific research. The benefits may be, in addition to direct income, shelter against natural phenomena, and the contribution of elements for activities or practices of a medicinal, religious or aesthetic nature (CONABIOINE, 1998).

Of the 78 species currently present in the region, 26 have some type of use, representing $33 \%$ of the total; by group, the percentages of use are $46 \%$ for birds, $19 \%$ for mammals and $35 \%$ for reptiles, that is, a third of the wildlife population living in the region is being captured for five different uses: pet (UV $\left.{ }_{\text {pet }}\right)$, food $\left(U V_{\text {food }}\right)$, medicinal $\left(U V_{\text {med }}\right)$, ritual $\left(U V_{\text {rit }}\right)$ and escala se determinó en cuatro valores, que van desde alto hasta muy escaso; del total de especímenes, solo $14 \%$ registró una abundancia alta, en el caso de los reptiles $42 \%$ se reporta como escaso e incluso, $17 \%$ de mamíferos se percibe como muy escaso, además de que este grupo es el que muestra mayor reporte de escasez. El Cuadro 3 presenta mayor detalle de los resultados por grupo.

De acuerdo con Santos-Fita, ArguetaVillamar, Astorga-Domínguez, y QuiñonezMartínez (2012), los vínculos cognitivos, emocionales y comportamentales que se establecen entre el ser humano y la fauna que lo rodea, definen al hombre mismo, ya sea por el valor tangible de la última o por su relevancia ecológica. Es por ello, que resulta necesario conocer no solo la percepción, sino además la relación que se desarrolla y las actitudes hacia las distintas especies. En este proyecto se cuestionó a los entrevistados sobre su reacción ante los diferentes especímenes identificados, los resultados para algunos de ellos, especialmente para la mayoría de los mamíferos fue de indiferencia en los tres grupos encuestados. Sin embargo, se 
ornamental $\left(\mathrm{UV}_{\mathrm{or}}\right)$. Birds are the most frequently used as pets and reptiles as food.

Table 4 shows the different indicators for each of the species, of which the crocodile stands out in the general use index, with three of the five uses detected, in addition to serving as food. It is believed that the crocodile's fat serves to relieve rheumatic discomfort and its teeth are sold to make jewelry. For the buff-bellied hummingbird and the ferruginous pygmy owl, ritual uses have been detected, as the belief in paranormal phenomena is deeply rooted throughout Mexico, especially in communities in the south of the country. According to Díaz-Rosales (2011), witchcraft uses prayer and supplication to restore or break equilibrium (during an illness, for example). Today, witchcraft's repertoire is broad and can produce or cure various issues such as lovesickness, caída de la mollera (Western medicine refers to this serious illness in infants as a sunken fontanelle, or a depression in the skull where the bones have not yet grown together and hardened), evil eye, mal de espanto (a disease suffered by humans, especially children, which can lead to the loss of one's soul as a result of a great shock or deep fear) and empacho (bloating caused by excessive eating), among others. In the case of the buff-bellied hummingbird, recognized as the bird of love, the different traditional healers of the locality (four people were identified who engage in these practices only in the town of Loma Bonita) ask their clients to get a hummingbird to be able to carry out the corresponding spell. On the other hand, the ferruginous pygmy owl has a symbolic function, as it is recognized for its wisdom and for being a very active guardian. They are found both alive and dissected in places where witchcraft is practiced. The difference between these practices and medicinal ones (for which other species such as the different varieties of turtle and skunk are used) is that the rituals are performed only by people to whom certain special knowledge is attributed, while the use of blood, meat or fat is made by the general population; for example, the turtle is killed and its blood drawn that, while hot, should be consumed to relieve chronic respiratory diseases.

According to the results, reptiles have higher Frequency of Use (FEU) rates, indicating that more people are engaged in their capture, explained in presentan diferencias significativas en la reacción de los diferentes grupos hacia algunos ejemplares del grupo de las aves y los reptiles, por lo que se realizó un análisis de correspondencias. Los resultados muestran una asociación entre el comportamiento y el grupo al que pertenecen (prueba Chi-cuadrada, $P<0.0001)$, siendo los productores quienes hacen mayor uso de la fauna (Figuras 1 y 2 ).

\section{Resultados sobre indicadores de valor de uso}

Al presentarse como satisfactores de necesidades humanas, los recursos naturales se convierten en elementos útiles, entendiendo como utilidad o uso, aquellas actividades a través de las cuales los seres humanos obtienen algún beneficio, ganancia, fruto o disfrute de una población animal. Los usos pueden ser directos o indirectos, consuntivos o no consuntivos (Alcérreca, et al., 1988). Las actividades pueden ser personales o familiares (cuando se utilizan para consumo familiar); comerciales (cuando se intercambian en un mercado) o de otro carácter como la educación e investigación científica. Los beneficios pueden ser, además de los ingresos directos, el abrigo contra fenómenos naturales, el aporte de elementos para actividades o prácticas de índole medicinal, religiosa o bien estéticas (CONABIO-INE, 1998).

De las 78 especies con presencia actual en la región, 26 tienen algún tipo de uso, lo que representa $33 \%$ del total; por grupo, los porcentajes de uso resultan en $46 \%$ para aves, $19 \%$ para mamíferos y $35 \%$ para reptiles, es decir, un tercio de la población de la fauna silvestre que habita en la región está siendo capturada para cinco distintos usos; mascota $\left(\mathrm{VU}_{\text {mas }}\right)$, alimento $\left(\mathrm{VU}_{\text {ali }}\right)$, medicinal $\left(\mathrm{VU}_{\text {med }}\right)$, ritual $\left(\mathrm{VU}_{\text {rit }}\right)$ y ornamental $\left(\mathrm{VU}_{\mathrm{or}}\right)$. Siendo las aves las más frecuentes como mascotas y los reptiles como alimento.

En el Cuadro 4, se presentan los diferentes indicadores para cada una de las especies, de los cuales destaca en el índice general de uso el cocodrilo o lagarto, con tres de los cinco usos detectados, además de servir como alimento, se cree que la grasa de este sirve para aliviar malestares reumáticos y los colmillos son vendidos para hacer bisutería. Para el colibrí y el tecolote, se han detectado usos denominados como rituales, la 
Table 4. Use indicators.

Cuadro 4. Indicadores de uso.

\begin{tabular}{|c|c|c|c|c|c|c|c|}
\hline $\begin{array}{l}\text { Common name / } \\
\text { Nombre común }\end{array}$ & $\begin{array}{l}\mathrm{GUI}_{\mathrm{x}} / \\
\mathrm{IGU}_{\mathrm{x}}\end{array}$ & $\begin{array}{l}\text { FEU }_{\mathbf{x}} / \\
\operatorname{FUE}_{\mathbf{x}}\end{array}$ & $\begin{array}{l}\mathbf{U V}_{\text {pet }} I \\
\mathbf{V U}_{\text {mas }}\end{array}$ & $\begin{array}{l}\mathbf{U V}_{\text {food }} I \\
\mathbf{V U}_{\text {ali }}\end{array}$ & $\begin{array}{l}\mathrm{UV}_{\text {med }} I \\
\mathrm{VU}_{\text {med }}\end{array}$ & $\begin{array}{l}\mathbf{U V}_{\text {rit }} / \\
\mathbf{V U}_{\text {rit }}\end{array}$ & $\begin{array}{l}\mathbf{U V}_{\text {or }} / \\
\mathbf{V u}_{\text {or }}\end{array}$ \\
\hline $\begin{array}{l}\text { Great kiskadee / } \\
\text { Pecho amarillo }\end{array}$ & 0.2 & 0.3 & 0.07 & & & & \\
\hline $\begin{array}{l}\text { Baltimore oriole / } \\
\text { Oropéndola }\end{array}$ & 0.2 & 0.2 & 0.03 & & & & \\
\hline $\begin{array}{l}\text { Ringed kingfisher / } \\
\text { Martín pescador de } \\
\text { collar }\end{array}$ & 0.2 & 0.3 & 0.06 & & & & \\
\hline $\begin{array}{l}\text { Ferruginous } \\
\text { pygmy owl / } \\
\text { Tecolote }\end{array}$ & 0.2 & & & & & 1 & \\
\hline $\begin{array}{l}\text { Blue-gray tanager / } \\
\text { Azulejo }\end{array}$ & 0.2 & 0.4 & 0.08 & & & & \\
\hline $\begin{array}{l}\text { Buff-bellied } \\
\text { hummingbird / } \\
\text { Colibrí }\end{array}$ & 0.4 & 0.4 & 0.08 & & & 1 & \\
\hline Pepencha & 0.2 & 0.3 & & 0.05 & & & \\
\hline $\begin{array}{l}\text { Golden-fronted } \\
\text { woodpecker / } \\
\text { Pájaro carpintero }\end{array}$ & 0.2 & 0.3 & 0.07 & & & & \\
\hline Cooper's hawk / Gavilán & 0.2 & 0.3 & 0.05 & & & & \\
\hline $\begin{array}{l}\text { Red-lored amazon / } \\
\text { Cotorro }\end{array}$ & 0.2 & 1.0 & 0.21 & & & & \\
\hline $\begin{array}{l}\text { Olive-throated } \\
\text { parakeet / } \\
\text { Cotorritos }\end{array}$ & 0.2 & 0.9 & 0.20 & & & & \\
\hline $\begin{array}{l}\text { Hooded warbler / } \\
\text { Chipe encapuchado }\end{array}$ & 0.2 & 0.2 & 0.04 & & & & \\
\hline $\begin{array}{l}\text { Nine-banded } \\
\text { armadillo / } \\
\text { Armadillo - toche }\end{array}$ & 0.2 & 0.5 & & 0.07 & & & \\
\hline Raccoon / Mapache & 0.4 & 0.2 & 0.04 & & & & 1 \\
\hline Hooded skunk / Zorrillo & 0.2 & 0.1 & & & 0.06 & & \\
\hline $\begin{array}{l}\text { Northern tamandua / } \\
\text { Oso hormiguero }\end{array}$ & 0.2 & 0.3 & 0.07 & & & & \\
\hline $\begin{array}{l}\text { Mexican cottontail / } \\
\text { Conejo silvestre }\end{array}$ & 0.2 & 0.4 & & 0.06 & & & \\
\hline $\begin{array}{l}\text { Mexican spiny-tailed } \\
\text { iguana / } \\
\text { Iguana negra, garrobo }\end{array}$ & 0.2 & 0.6 & & 0.09 & & & \\
\hline $\begin{array}{l}\text { Red-eared slider / } \\
\text { Tortuga pinta orejas } \\
\text { rojas }\end{array}$ & 0.2 & 0.6 & & 0.12 & & & \\
\hline $\begin{array}{l}\text { Morelet's crocodile / } \\
\text { Cocodrilo }\end{array}$ & 0.6 & 0.4 & & 0.05 & 0.20 & & 1 \\
\hline
\end{tabular}


Table 4. Use indicators. (cont.)

Cuadro 4. Indicadores de uso. (cont.)

\begin{tabular}{|c|c|c|c|c|c|c|c|}
\hline $\begin{array}{l}\text { Common name / } \\
\text { Nombre común }\end{array}$ & $\begin{array}{l}\text { GUI }_{x} I \\
\text { IGU }_{x}\end{array}$ & $\begin{array}{l}\text { FEU }_{x} / \\
\text { FUE }_{x}\end{array}$ & $\begin{array}{l}\mathbf{U V}_{\text {pet }} I \\
\mathbf{V U}_{\text {mas }}\end{array}$ & $\begin{array}{l}\mathrm{UV}_{\text {food }} I \\
\mathbf{V U}_{\text {ali }}\end{array}$ & $\begin{array}{l}\mathbf{U V}_{\text {med }} I \\
\mathbf{V U}_{\text {med }}\end{array}$ & $\begin{array}{l}\mathbf{U V _ { \text { rit } }} / \\
\mathbf{V u}_{\text {rit }}\end{array}$ & $\begin{array}{l}\mathbf{U V}_{\text {or }} / \\
\mathbf{V u}_{\text {or }}\end{array}$ \\
\hline $\begin{array}{l}\text { Common snapping } \\
\text { turtle / } \\
\text { Tortuga lagarto }\end{array}$ & 0.4 & 0.3 & & 0.08 & 0.20 & & \\
\hline $\begin{array}{l}\text { Mexican musk turtle / } \\
\text { Tortuga tres lomos }\end{array}$ & 0.4 & 0.5 & & 0.10 & 0.20 & & \\
\hline Green iguana / Iguana & 0.2 & 0.6 & & 0.08 & & & \\
\hline $\begin{array}{l}\text { Narrow-bridged musk } \\
\text { turtle / } \\
\text { Tortuga chopontil }\end{array}$ & 0.4 & 0.5 & & 0.13 & 0.33 & & \\
\hline $\begin{array}{l}\text { Meso-American slider / } \\
\text { Tortuga pinta }\end{array}$ & 0.4 & 0.8 & & 0.11 & 0.20 & & \\
\hline $\begin{array}{l}\text { White-lipped } \\
\text { mud turtle / } \\
\text { Tortuga casquito }\end{array}$ & 0.4 & 0.7 & & 0.10 & 0.20 & & \\
\hline
\end{tabular}

Source: Author-made.

Fuente: Elaboración propia.

part by the ease of obtaining them; the contrast can be seen with specimens that require greater effort to capture them, such as Morelet's crocodile or the narrow-bridged musk and common snapping turtles. Of the birds, parrots and parakeets, in addition to having a good market price, are relatively easy to capture because it is done when they are newborns.

\section{Results on the economic valuation}

In the town of Loma Bonita, in general, the capture of these animals is done opportunistically and is carried out, for the most part, by people who are engaged in agricultural work, that is, if during their daily activities they detect any specimen, they suspend their labors and try to capture the animal; once they have caught and secured the animal, they continue with their activity and at the end of the day they have it up for sale. Although most are consumers, especially of the species that serve as food, the main intention of hunting, for most species, is to sell the animal in the local market; if after a while they cannot sell it, then they decide to consume it with their family, although they usually do not have trouble selling it. Marketing is carried out by word of mouth, in such a way that the only thing they have to creencia en fenómenos de carácter paranormal está profundamente arraigada en todo México, especialmente en comunidades del sur de país. De acuerdo con Díaz-Rosales (2011), la brujería emplea la oración y la súplica para restablecer o romper el equilibrio (durante una enfermedad, como ejemplo). En la actualidad el repertorio de la brujería es amplio y puede producir o curar diversas cuestiones tales como tiricia o mal de amores, caída de la mollera, mal de ojo, mal de espanto y empacho entre otros. En el caso del colibrí, reconocido como el pájaro del amor, los diferentes brujos de la localidad (se identificaron a cuatro personas que se dedican a estas prácticas solo en la localidad de Loma Bonita) solicitan a los clientes que consigan un colibrí para poder realizar el hechizo correspondiente. El tecolote tiene más bien la función de simbolismo, ya que se les reconoce por su sabiduría y por ser guardianes muy activos, se les observa tanto vivos como disecados en lugares donde se práctica la brujería. La diferencia de estas prácticas con las medicinales, (para las cuales son usadas otras especies tales como las diferentes variedades de tortuga y el zorrillo), es que los rituales son realizados solo por personas a las que se les atribuyen ciertos conocimientos especiales, mientras 
do is to comment that they have some species for sale and the buyer usually comes to their home to carry out the transaction. Some species are usually sold on demand, especially those for medicinal or ritual uses.

In order to estimate the cost-benefit of each of the species (Table 5), the effort involved in catching, slaughtering or processing market products was evaluated. These costs are very low as not many hunting implements are used. Some species such as the crocodile require more strategy and strength, and it is one of the few specimens that should be fed and kept at home until it reaches a larger size, que el uso de sangre, carne o grasa se hace por parte de la población en general; por ejemplo la tortuga se mata y se le extrae la sangre que mientras esté caliente debe consumirse para aliviar enfermedades respiratorias crónicas.

De acuerdo con los resultados, los reptiles presentan mayores índices de Frecuencia de Uso (FUE), lo que indica que un mayor número de personas se dedican a su captura, explicado en parte, por la facilidad para conseguirlas; puede verse el contraste con especímenes que requieren mayor esfuerzo, como el cocodrilo o las tortugas chopontil y lagarto. De las aves, los cotorros y cotorritos, además

Table 5. Economic value of species.

Cuadro 5. Valor económico de las especies.

\begin{tabular}{|c|c|c|c|}
\hline Common name / Nombre común & $\begin{array}{l}\text { U. of measure / } \\
\text { U. de medida }\end{array}$ & $\begin{array}{l}\text { Price }_{x} / \\
\text { Precio }_{x}\end{array}$ & $\begin{array}{l}\text { C/B / } \\
\text { B/C }\end{array}$ \\
\hline $\begin{array}{l}\text { Great kiskadee, buff-bellied hummingbird, } \\
\text { golden-fronted woodpecker, hooded warbler / } \\
\text { Pecho amarillo, colibrí, pájaro carpintero, chipe }\end{array}$ & Specimen / Ejemplar & 300 & 6 \\
\hline Baltimore oriole / Oropéndola & Specimen / Ejemplar & 550 & 11 \\
\hline $\begin{array}{l}\text { Ringed kingfisher, Cooper's hawk / } \\
\text { Martín pescador de collar, gavilán }\end{array}$ & Specimen / Ejemplar & 400 & 8 \\
\hline Ferruginous pygmy owl / Tecolote & Specimen / Ejemplar & 1000 & 5 \\
\hline $\begin{array}{l}\text { Blue-gray tanager, olive-throated parakeet / } \\
\text { Azulejo, cotorritos }\end{array}$ & Specimen / Ejemplar & 500 & 10 \\
\hline Red-lored amazon / Cotorro & Specimen / Ejemplar & 700 & 14 \\
\hline Nine-banded armadillo / Armadillo/toche & Specimen / Ejemplar & 400 & 4 \\
\hline Raccoon, hooded skunk / Mapache, zorrillo & Kilogram / Kilogramo & 300 & 3 \\
\hline Raccoon skin / Piel de mapache & Skin / Piel & 70 & 7 \\
\hline Northern tamandua / Oso hormiguero & Specimen / Ejemplar & 700 & 7 \\
\hline Mexican cottontail / Conejo silvestre & Specimen / Ejemplar & 60 & 6 \\
\hline $\begin{array}{l}\text { Mexican spiny-tailed iguana, green iguana / } \\
\text { Iguana negra, garrobo, iguana }\end{array}$ & Specimen / Ejemplar & 150 & 5.6 \\
\hline $\begin{array}{l}\text { Red-eared slider, Mexican musk turtle, Meso- } \\
\text { American slider, white-lipped mud turtle/ } \\
\text { Tortugas: pinta orejas rojas, tres lomos, } \\
\text { pinto, casquito }\end{array}$ & Specimen / Ejemplar & 200 & 7.4 \\
\hline Morelet's crocodile / Cocodrilo & Kilogram / Kilogramo & 100 & 2 \\
\hline Crocodile fat / Grasa de cocodrilo & Liter / Litro & 400 & 8 \\
\hline Crocodile teeth / Dientes de cocodrilo & Specimen / Ejemplar & 80 & 4 \\
\hline $\begin{array}{l}\text { Common snapping and narrow-bridged } \\
\text { musk turtles / } \\
\text { Tortuga lagarto y chopontil }\end{array}$ & Specimen / Ejemplar & 350 & 3.5 \\
\hline
\end{tabular}

Source: Author-made.

Fuente: Elaboración propia. 
since there is a direct relationship between the weight and amount of production, as well as the size of the teeth. Crocodiles together with parrots are the most profitable species since several products are obtained from the same specimen. It should be noted that, for producers, who do not count their work as a real cost, the perception of profit is much broader, since the market price is seen as a net benefit.

\section{Discussion}

According to Lagunas-Vazques, Beltrán-Morales, Santiago-León, and Ortega-Rubio (2013), 175 protected natural areas have been identified in Mesoamerica that share space with around 80 diverse cultures, so the emphasis on protection should not only focus on flora and fauna, but also on the survival of these populations as in situ managers and producers of the environment. The concept of symbiotic conservation or biocultural axiom should be broadly researched and legislated upon.

The present study has described the use and importance of wildlife in the community of Loma Bonita, providing theoretical-technical elements that should be considered in the event that local-state authorities could develop them into a conservation program, especially for species that could be at greater risk because they are under pressure due to the uses given to them.

This knowledge, together with the perception of abundance-scarcity of the inhabitants themselves, is fundamental in strategy proposals at the regional and national levels. Although the lack of absolute measures of abundance is recognized as a limiting factor in this study, studies conducted by authors such as Alemán-Mejía (2011) and Suárez-Islas (2008) found great similarity between the real abundance of species (reptiles in the first case and flora in the second) and the perception of abundance measured by the local population.

Another element that should not be ignored is the economic valuation of community species. In this regard, we agree with Pearce and Turner (1990) when they point out that the greater the awareness of both the supply and demand of the value of the natural resource, the greater its market value and, therefore, the reasons for preserving it. de que tienen buen precio de mercado, su captura también es relativamente fácil ya que se realiza cuando son recién nacidos.

\section{Resultados sobre la valoración económica}

En la localidad de Loma Bonita, por lo general, la captura de estos animales es de oportunidad y se realiza, en su mayoría, por personas que se dedican a labores agropecuarias, esto es, si durante sus actividades diarias detectan algún ejemplar, la suspenden y van a la captura del animal, una vez asegurada su presa, siguen con su actividad y al final de la jornada la disponen para su venta. Aunque la mayoría es consumidora, especialmente de las especies que sirven como alimento, la intención principal de la cacería es la venta en el mercado local - para la mayoría de las especies-si pasado un tiempo no logran colocarla, entonces deciden consumirla familiarmente, aunque generalmente no suelen tener problemas para venderla. La comercialización se realiza de voz en voz, de tal manera que lo único que tienen que hacer es comentar que tienen alguna especie a la venta y el comprador llega por lo regular hasta sus casas para realizar la transacción. Algunas especies, habitualmente se comercializan por pedido, especialmente las dedicadas a usos medicinales o rituales.

Para estimar el beneficio-costo de cada una de las especies (Cuadro 5), se recurrió a la valoración del esfuerzo que implica la captura, matanza o procesamiento de los productos de mercado. Estos costos resultan realmente bajos ya que no se utilizan muchos implementos de caza, algunas especies como el cocodrilo requieren más estrategia y fuerza, además de que es de los pocos ejemplares que conviene alimentar y mantener en casa hasta alcanzar un tamaño más grande, ya que existe una relación directa entre el peso y la cantidad de producción, así como el tamaño de los colmillos. Siendo esta junto con los cotorritos la especie más rentable ya que se obtienen varios productos de un mismo ejemplar. Cabe señalar que, para los productores, quienes no contabilizan su trabajo como un costo real, la percepción de ganancia es mucho más amplia, ya que el precio de mercado se aprecia como un beneficio neto. 
Finally, this research has shown, in terms of perception, the disappearance of 10 wildlife species that residents are currently very aware of, so it could be an important motivation in the conservation of species. The fact that responsibility for their disappearance is assumed, even if only partially, by the inhabitants and that a major effort is also perceived as being necessary for the capture of the species, constitutes an argument that should be continually pointed out.

End of English version

\section{References / Referencias}

Adams B., Berkes F., Ferreira de Athayde S., Dudley N., Hunn E., Maffi L.,... S. Pilgrim. (2008). ¿How Do Biodiversity and Culture Intersect? Plenary paper for Conference "Sustaining Cultural and Biological Diversity In a Rapidly Changing World: Lessons for Global Policy". Organized by American Museum of Natural History's Center for Biodiversity and Conservation, IUCN-The World Conservation Union/Theme on Culture and Conservation, and Terralingua. April 2-5th 2008 Reyes, J.A., J.P. Gómez, R.O. Muis, R. Zavala, G.A. Ríos y O. Villalobos. 2012.

Aguilar-Barojas, S. (2005). Fórmulas para el cálculo de la muestra en investigaciones de salud en Tabasco, vol. 11, núm. 1-2, enero-agosto, pp. 333-338.

Alcérreca C., Consejo J., Flores O., Gutiérrez D., Hentschel E., Herzig M.,... Sanchez-Cordero V. (1988). Fauna silvestre y áreas naturales protegidas. Colección Medio México. Ambiente, núm. 7. Fundación Universo XXI.

Alemán-Mejía, J. (2011). Caracterización de reptiles y percepción local hacia las serpientes en fincas ganaderas de la subcuenca del Río Copán, Honduras. Agroforestería en las Américas, 48, 103-117.

Alexiades, N. (1996). Selected Guidelines For Ethnobotanical Research: A Field Manual. Scientific Publications. Department. New York Botanical Garden. New York.

Alfaro, A. M., García-García, J. L., \& Santos-Moreno, A. (2006). Mamíferos de los municipios Santiago Jocotepec, Ayotzintepec, Chinantla Baja, Oaxaca. Naturaleza y Desarrollo 4(1):19-23.

\section{Discusión}

De acuerdo con Lagunas-Vazques, BeltránMorales, Santiago-León, y Ortega-Rubio (2013), en Mesoamérica se identifican 175 áreas naturales protegidas que comparten espacio con alrededor de 80 culturas diversas, por lo que el énfasis en la protección no solo debiera enfocarse a la flora y fauna, sino también en la supervivencia de estas poblaciones como manejadoras, gestionadoras y productoras in situ del ambiente. En un concepto amplio debe investigarse y legislarse sobre el concepto de conservación simbiótica o axioma biocultural.

El presente estudio ha descrito el uso e importancia de la fauna silvestre en la comunidad de Loma Bonita, aportando elementos teórico-técnicos que deberían ser contemplados en caso de que las autoridades locales-estatales pudieran cristalizarse en algún programa de conservación. En especial, para las especies que podrían presentar mayor riesgo al estar bajo presión por los usos que se les da.

Este conocimiento, aunado a la percepción de abundancia-escasez de los mismos pobladores, es fundamental en la propuesta de estrategias a nivel regional y nacional. Aun y cuando se reconoce como limitante en este trabajo la falta de medidas absolutas de la abundancia, estudios presentados por autores como Alemán-Mejía (2011) y SuárezIslas (2008), encontraron gran similitud entre la abundancia real de las especies (reptiles en el primer caso y flora en el segundo) y la percepción de la abundancia medida por la población local.

Otro elemento que no debe descartarse es el de la valoración económica de las especies de la comunidad. En este aspecto coincidimos con Pearce y Turner (1990), cuando señala que entre mayor conciencia se tenga, tanto de la oferta como de la demanda del valor del recurso natural, mayor será su valor de mercado $y$, por tanto, los motivos para preservarlo.

Finalmente, la investigación ha evidenciado en términos de percepción, la desaparición de 10 especies de fauna que hoy en día los pobladores tienen muy presente, por lo que podría ser una motivación importante en la conservación de especies. El hecho de que se asuma, aunque sea 
Álvarez Castañeda, S. (2003). Ficha técnica de Procyon insularis. Roedores y carnívoros del noroeste de México incluidos en el Proyecto NOM-059-ECOL-2001. Centro de Investigaciones Biológicas del Noroeste S.C. Bases de datos SNIB-CONABIO. Proyecto No. W003. México, D.F.

Ávila-Nájera D., Rosas-Rosas O., Tarango-Arámbula L., Martínez-Montoya J., \& Santoyo-Brito E. (2011). Conocimiento, uso y valor cultural de seis presas del jaguar (Panthera onca) y su relación con éste, en San Nicolás de los Montes, San Luis Potosí, México. Revista Mexicana de Biodiversidad 82: 1020-1028.

Badii, M., Castillo, J., Cortez, K., Wong, A., \& Villalpando, P. (2007). Análisis de correlación canónica (ACC) e investigación científica Innovaciones de Negocios 4(2): 405 - 422, UANL, Impreso en México (ISSN 1665-9627)

Bennett N., \& Dearden, P. (2013a). Why local people do not support conservation: Community perceptions of marine protected area livelihood impacts, governance and management in Thailand. Marine Policy 44:107-116.

Bezaury-Creel, J., Gutiérrez-Carbonell, D., \& Remolina, J. F. (2009). Áreas naturales protegidas y desarrollo social en México, en Capital natural de México, vol. II: Estado de conservación y tendencias de cambio. Conabio, México, pp. 385-431.

Boege, E. (2009). El reto de la conservación de la biodiversidad en los territorios de los pueblos indígenas, En: Dirzo, R., González, R., \& March, I. J. Capital natural de México, vol. II: Estado de conservación y tendencias de cambio. Conabio, México, pp. 603-649.

Buena-Zepeda, J., Castillo-Romano, C., Castillo-Campos, G., Pale-Pale, J., \& Moreno-Casasola, P. (2012). Inventario y caracterización de humedales en la cuenca del río Papaloapan, México. Tecnología y ciencias del Agua, 3(2):131.139.

Campbell, J. (1998). Amphibians and Reptiles of Northern Guatemala, The Yucatán and Belize. University of Oklahoma Press. Oklahoma, U.S.A.

Casas-Andreu, G., Méndez-de la Cruz, F., \& Camarillo, J. L. (1996). Anfibios y reptiles de Oaxaca. Acta Zoológica Mexicana.

Comisión Nacional para el Conocimiento y Uso de la Biodiversidad (CONABIO). (2000). Humedales del Papaloapan, San Vicente y San Juan.

Comisión Nacional para el Conocimiento y Uso de la Biodiversidad/Instituto Nacional de Ecología. (CONABIO-INE). (1998). Aspectos económicos sobre la biodiversidad de México. México. parcialmente, la responsabilidad de su desaparición, y que además se perciba un mayor esfuerzo en la captura de las especies, constituye un argumento sobre el que se deba seguir puntualizando.

Fin de la versión en español

Cruz-Espinoza, A., González-Pérez, G., \& Ronel-Vázquez, P. (2012). Nota de la variación en la riqueza específica de mamíferos entre áreas de conservación y de aprovechamiento forestal en la Sierra Madre de Oaxaca. THERYA 3(3):327-332.

Davidson-Hunt, I. J., Suich, H., Meijer, S. S., \& Olsen, N. (eds.). (2016). People in Nature: Valuing the diversity of interrelationships between people and nature. Gland, Switzerland: IUCN. Gland, Switzerland: IUCN. 108pp.

Díaz-Rosales, J. (2011). Mitos y ciencia: Brujería, herbolaria y autosugestión. http://www.medigraphic.com/pdfs/ bmhfm/hf-2011/hf111g.pdf Consultado el 12/08/2018.

Dixon, J., Scura, L., Carpenter, R., \& Sherman P. (1994). Economic Analysis of Environmental Impacts. London: Earthscan

Durand, L., \& Jiménez, J. (2010). Sobre áreas naturales protegidas y la construcción de nolugares: Notas para México. Revista Lider 16(12): 59-72.

Figueroa-Solano, E. (2000). Uso agroecológico, actual y potencial, de especies arbóreas en una selva baja caducifolia perturbada del suroeste del Estado de México. Tesis, Maestría Colegio de Postgraduados, Montecillo, Texcoco, Estado de México. 38-39 p.

Flores-Villela, O. A. (1993). Lista anotada de las especies de anfibios y reptiles de México, cambios taxonómicos recientes, y nuevas especies. The Carnegie Museum of Natural History. Pittsburgh, U.S.A.

Gese, E. M., Bekoff, M., Andelt, W., Carbyn, L., \& Knowlton, F. (2008). Canis latrans. Lista Roja de Especies Amenazadas IUCN. Versión 2010.1. <www.iucnredlist. org >. Consultado el 19 de abril de 2010.

Gómez-Nísino, A. (2006). Ficha técnica de Lontra longicaudis. En: Medellín, R. (compilador). Los mamíferos mexicanos en riesgo de extinción según el PROY-NOM-059-ECOL-2000. Instituto de Ecología, Universidad Nacional Autónoma de México. Bases de datos SNIB-CONABIO. Proyecto No. W005. México, D.F.

Hernández-Xolocotzi, E. (1977). Ed. Agroecosistemas de México: contribuciones a la enseñanza, investigación 
y divulgación agrícola. Colegio de Postgraduados. Chapingo, Estado de México. 559 p. Segunda edición 1981.

Hotelling, H. (1935). The most predictable criterion. Journal of Educational Psychology. 26(2): 139-142.

Jiménez-Ferrer, G., Velasco-Pérez, R., Uribe-Gómez, M., \& Soto-Pinto I. (2008). Ganadería y conocimiento local de árboles y arbustos forrajeros de la selva Lacandona, Chiapas, México. Zootecnia Tropical 26 (3):333-337.

Kido, A., \& Seidl, A. (2008). Optimizing protected area entry fees across stakeholders: The monarch butterfly biosphere reserve; Michoacan, Mexico. Environment and Development Economics. 13:229-243.

Lagunas-Vazques, M., Beltrán-Morales, L. F., SantiagoLeón, F., \& Ortega-Rubio, A. (2013). Indicadores sociales: desarrollo humano en la reserva de la biosfera Sierra La Laguna. En: M. Lagunas-Vázques, L. F. Beltrán-Morales y A. Ortega-Rubio. (Editores).

Mexican Association for Conservation and Study of Lagomorphs (AMCELA), Romero Malpica, F. J., \& Rangel Cordero, H. (2008). "Sylvilagus cunicularius". IUCN Red List of Threatened Species. Version 2010.4. International Union for Conservation of Nature. Retrieved 24 Feb. 2011.

Naturalista, CONABIO http://www.naturalista.mx Consultado el 06/03/2016.

Newbold, P., Carlson, W., \& Thorne B. (2008). Estadística para Administración y Economía. Sexta edición. Thorne Stetson University. Traducción, Esther Rabasco Espáriz, Revisión Técnica Luis Toharia, Universidad de Alcalá de Henares Prentice Hall, Upper Saddle River, New Jersey 07458 Madrid.

Pearce, D. W., \& Turner R., K. (1990). Economics of natural resources and the environment. Harvester Wheat Sheaf, London, UK.

Pérez-Higareda, G., \& Smith, H. (1991). Ofidio fauna de Veracruz. Análisis taxonómico y Zoogeográfico. Instituto de Biología, UNAM. México.

Pérez-Irineo, G., \& Santos-Moreni, A. (2012). Diversidad de mamíferos terrestres de talla grande y media de una selva subcaducifolia del noreste de Oaxaca, México. Revista Mexicana de Biodiversidad 83(1):164-169.

Pérez-Lustre, M., Contreras-Díaz, R. G., \& SantosMoreno A. (2006). Mamíferos del bosque mesófilo de montaña del municipio de San Felipe Usila, Tuxtepec, Oaxaca, México. Revista Mexicana de Mastozoología 10:29-40.
Phillips, O., \& Gentry, A. (1993). The Useful Plants of Tambopata, Peru: I Statistical Hypotheses Test with a New Quantitative Technique. Economic Botany. 47(1):15-32.

Puc G., \& Retana G. (2012). Uso de la fauna silvestre en la comunidad maya Villa de Guadalupe, Campeche, México. Etnobiología 10(2):1-11.

Ramírez-González, C., Lara-Brenis, A., \& Mijangos-Arrazola, T. (2014). New distributional records of amphibians and reptiles from northern Oaxaca, Mexico. 2014. Check List 10(3):679-681.

Ruitenbeek, H. (1990). Evaluating economic policies for promoting rainforest conservation in developing countries. PhD thesis, The London School of Economics and Political Science (LSE).

Ruiz-Velásquez, E., Andrés-Reyes, J., \& Santos-Moreno, A. (2014). Revista Mexicana de Biodiversidad 85(1):325-327.

Sánchez-Herrera, O., López-Segurajauregui, G., GarcíaNaranjo, A., \& Benítez-Díaz, H. (2011). Programa monitoreo del cocodrilo de pantano (Crocodylus moreletii) México-Belice-Guatemala. México: Comisión Nacional para el Conocimiento y Uso de la Biodiversidad.

Santos-Fita, D., Argueta Villamar, A., Astorga-Domínguez, M., \& Quiñonez-Martínez, M. (2012). La etnozoología en México: la producción bibliográfica del siglo XXI (20002011). Etnobiología 10(1).

Secretaría de Medio Ambiente y Recursos Naturales. México país megadiverso. https://www.gob.mx/ semarnat/articulos/mexico-pais-megadiverso-31976. Retrieved 11/03/2016.

Secretaría de Medio Ambiente y Recursos Naturales. (SEMARNAT). (2009.) Manual técnico para beneficiarios: Manejo de vida silvestre. Primera Edición (2009). Coordinación General de Educación y Desarrollo Tecnológico. Gerencia de Educación y Capacitación. Impreso en México.

Smith, H., \& Smith, R. B. (1976). Synopsis of the herpetofauna of Mexico. Soure Analysis and Index for Mexican Reptiles. John Johnson. III. Colorado, U.S.A.

Suárez, A., Palacios, C., Razo, R., Araujo R., \& Goche, J. (2011). Selección de especies leñosas para la restauración de ecosistemas forestales basada en el conocimiento campesino" In: Memorias del VII Congreso Mexicano de Recursos Forestales. Universidad Autónoma de Chihuahua. Chihuahua, Chihuahua. pp 545-546.

Suárez-Islas, A., (2008). Usando el conocimiento local en la selección de especies leñosas para restauración 
de bosques: análisis de dos experiencias en México.

Instituto de Ciencias Agropecuarias. Universidad Autónoma del Estado de Hidalgo. México.

Tamayo, J. L. (1977). Secretaría de Agricultura y Recursos Hidráulicos, México. Comisión del Papaloapan; Beltrán, E. Instituto Mexicano de Recursos Naturales Renovables. México.

Thiébaut, V. (2013). En torno al trabajo de campo en la cuenca baja del río Papaloapan. Un proyecto de investigación sobre paisajes fluviales e identidad. Revista de Investigaciones Geográficas, Universidad Nacional Autónoma de México, Instituto de Geografía. México.

Torres Colín. (2004). Tipos de vegetación. En: GarcíaMendoza, A.J., Ordóñez M.J., \& Briones-Salas, M. (eds.). Biodiversidad de Oaxaca. Instituto de Biología, UNAM-Fondo Oaxaqueño para la Conservación de la
Naturaleza-World Wildlife Fund, México, pp. 105-117.

Turner, N. (1988). The importance of a rose. Evaluating the cultural significance of plants in Thompson and Lillooet Interior Salish. American Anthropologist, new series 90:272-290.

Yang H., Rhett H., Zhuang-Fang Y., Goodale E., Ming-Xu Z., \& Jian-Chu, X. (2015). “Changing Perceptions of Forest Value and Attitudes toward Management of a Recently Established Nature Reserve: A Case Study in Southwest China. Forests 6(9): 3136-3164.

World Wildlife Fund (WWF). 2000. Indigenous and Traditional Peoples of the World and Ecoregion Conservation. Suiza. 120 pp.

Zertuche, J. J. (1981). Reptiles mexicanos de importancia para la salud pública y su distribución geográfica. Salud pública de México. XXIII (4):329-344. 
